\title{
Methods of deliberate self-harm in Pakistan
}

\author{
Murad Moosa Khan and Hashim Reza
}

\begin{abstract}
Reports of dellberate self-harm (DSH) are rare from Paklitan where poychotrople drugs can be obtained 'over the counter. A retroepective ancilyets of 382 index cases presenting to a untverstly hospltal in Karachi showed self-polsoning with benzodlezzepines as the moet common method, followed by organophosphate insecticides. Sallicylates and non-oplate analgestas were significantly absent from our series. Implications of these findinges ore discuseed.
\end{abstract}

Various studies have shown that poisoning by medication is the most common method in deliberate self-harm (DSH) (Hawton \& Fagg. 1992), and psychotropics, particularly benzodiazepines one of the most frequently used drug (Prescott \& Highley, 1985). Recently, high rates and increasingly frequent self-poisoning with analgesics, especially paracetamol have been reported (Hawton \& Fagg, 1992). Less than half the patients overdosing with medications have been found to use one drug only, whereas 20$37 \%$ used 3 or more drugs (Prescott \& Highley, 1985). The increased use of psychotropics has occurred in spite of the fact that in many countries prescription and dispensing of these drugs is strictly controlled. In many cases the drugs have been purchased with prescriptions issued to the self-poisoners (Alsen et al, 1994).

There have been few reports of DSH from countries like Pakdstan where most drugs, including psychotropics, are available 'over the counter'. A review of 1900 cases of acute poisoning admitted to a large government hospital in Karachi showed 'suicidal' attempts in $1330(70 \%)$ of the cases, of whom $40 \%$ used organophosphate insecticides, followed by 'tranquillisers' in 21\% (Jamil, 1990).

This study aims to give a descripttve account of the methods used by patients in their DSH episode. In cases of medication use, their source is also determined.

\section{The study}

A retrospective case note analysis was carried out of all index episodes of DSH referred to the psychiatry department of the Aga Khan Untversity Hospital (AKUH), Karachi, between January 1989 and July 1993.
Karachi is Pakistan's largest city, with an estimated population of over 10 million. The city has three major government hospitals apart from many small and medium sized private ones. The law in Pakistan requires every case of DSH to be reported to the police. This is done through medico-legal officers (MLOs) on duty in government hospitals. However, no prtvate hospitals in Pakdstan have MLOs. It is entirely on their discretion to report a certain case; almost none do so (Khan et al, 1995).

AKUH, located centrally in Karachi, has an excellent 24-hours emergency clinic for all spectalities, including psychiatry, but does not have a medico-legal section.

All patients presenting to the emergency room (ER) at AKUH after DSH are offered admission as per hospital policy. A small number of patients refuse admission for various reasons and discharge themselves from the ER. Those admitted are referred for a psychiatric evaluation before discharge. A log book of all DSH referrals is maintained and the medical records department also uses a separate code for these admissions. Both sources were used to retrieve the case notes for this study.

Data was compiled and analysed using the Epilnfo statistical package. Data for male and female groups were compared for differences using the $\chi^{2}$ test with Yates correction. Level of significance was taken at $P$ value of $<0.05$.

\section{Findings}

A total of 382 index cases of DSH were assessed by the psychiatry department during the study period. Females (59\%) outnumbered males (41\%) in a ratio of 1.41 . Their ages ranged from 12 to 76 years with a mean of 27.37 (s.d. $=10.48$ ); 262 $(69 \%)$ were under the age of 30 years.

Married women (32\%) formed the largest group. followed by single women (25\%), single men (24\%) and married men (16\%). The number of divorced and widowed individuals in both groups were negligible.

Medication overdose (73\%) was the most common method followed by organophosphate insecticides ingestion (20\%). Physical methods 
were used by $7 \%$. Among the medications, benzodiazepines as a single drug accounted for $60 \%$ of the cases but also featured in almost all cases of multiple drug overdose (5\%). Tricyclic antidepressants and antipsychotics each accounted for $4 \%$ of cases. There were no cases of salicylates, non-opiate analgesics, or alcohol use.

Of the drug overdoses ( $n=279$ ), $42 \%$ bought the drug over the counter. In $43 \%$ of cases the drugs were lying at home-either prescribed to the person previously but not being taken currently or belonged to another family member. Only $15 \%$ took an overdose of drugs prescribed to them. Gender was significantly assoclated with marital status $(P<0.05)$, methods of overdosing $(P<0.01)$ and benzodiazepine overdose $(P<0.005)$.

The majority of patients ( $80 \%)$, did not suffer from any medical complications of DSH and were discharged within three days of admission while $16 \%$ needed to stay for longer than five days. Fifteen per cent of patients were transferred to the psychiatric in-patient service. Three patients died, one of jumping from a height and two of insecticide poisoning.

\section{Comment}

Pakistan is a predominantly Muslim country where suicide and DSH are still considered criminal offences punishable by law. Islam strongly disapproves of such behaviour. This and the fact that there is considerable stigma to seeing a psychiatrist precludes many DSH cases from coming in contact with psychiatric services. Fearing persecution by police, many patients are taken to private hospitals which do not record such cases as DSH or attempted suicide. This would explain the seemingly low rate of DSH for the population size in this study.

Pakistani society is strongly patriarchal with women having few socially sanctioned ways of voicing their grievances or exerting their rights. The literacy rate is under $30 \%$ for males, and even lower for females. Analysis of our results show married women to be the largest group. This is in contrast to the findings of DSH studies from Europe and North America. Marriage appears to be a significant source of stress for women in Pakistan (Khan et al, 1995), as reflected in this study.

Studies of DSH from countries as diverse as Sweden (Alsen, 1994), Hong Kong (Chan, 1994) and the United States (Stein, 1993) show that the majority of patients use medications, particularly benzodiazepines, in their suicide attempt. These countries differ from Pakistan in many areas but notably in their religious and cultural beliefs, literacy rates and legal issues relating to suicide and DSH. Despite such differences our findings have many similarities with these countries.
Poisoning by medication was the most common method, with benzodiazepines being the most commonly used drug. However, salicylates and non-opiate analgesics were completely absent though these medicines are as easy to procure as benzodiazepines.

Although benzodiazepines (especially temazepam), have been implicated in fatal poisonings, compared to other psychotropics such as tricyclic antidepressants and paracetamol they are safer in overdosage. On the other hand poisoning with organophosphate insecticides has more serious outcomes. In Jamil's (1990) series of acute poisoning cases, these substances were responsible for $67 \%$ of the 107 fatalities. They are found commonly in most Pakistani households and their easy accessibility would account for the high rate seen in our studies.

The preference for benzodiazepines over analgesics in this study may be due to the common perception in Pakistan of the former being 'sleeping pills' (sleep being equated with 'loss of consciousness') while analgesics being 'pain killers' may not give the 'mental relief desired by many of these patients.

\section{Acknowiedgement}

Our thanks to the personnel at the Medical Records Department at AKUH for their invaluable help.

\section{References}

ALSEN, M., EKEDAHL, A., LOWENHELM, P., et al (1994) Medicine self poisoning and the sources of drugs in Lund. Sweden. Acta Psychiatrica Scandinavica, 89, 255-261.

Chan, T. Y., CrTrchleY, J. A., Chan, M. T., et al (1994) Drug overdosage and other polsoning in Hong Kong - the Prince of Wales Hospital (Shatin) experience. Human and Experimental Taxicology, 23, 512-515.

HAwTON, K. \& FAGG, J. (1992) Trends in deliberate self poisoning and self injury in Oxford, 1976-90. British Medical Journal, SO4, 1409-1411.

JAMI, H. (1990) Acute poisoning - a review of 1900 cases. Joumal of Pakistan Medical Assoctation, 40, 131-133.

KHAN, M. M., IsLAM, S. \& KUNDI, A. (1996) Parasuicide in Pakdstan: experience at a untversity hospital. Acta Psychiatrica Scandinavica, 83 (in press).

PrescoTt, L. P. \& HighLEY, M. S. (1985) Drugs prescribed for self polsoners. Brttish Medical Joumal, 290, 1633-1636.

Stein, M. D., Bonanno, J., O'Suluvan, P. S., et al (1993) Changes in pattern of drug overdoses. Journal of General Internal Medictne, 8, 179-184.

*Murad Moosa Khan, Assistant Professor; and Hashim Reza, Assistant Professor, Department of Psychiatry, The Aga Khan University Hospital, PO Bax 3500, Stadium Road, Karachi 74800, Pakistan

*Correspondence 